\title{
Correction to: Histone methyltransferase WHSC1 inhibits colorectal cancer cell apoptosis via targeting anti-apoptotic BCL2
}

Yu Wang, Liming Zhu, Mei Guo, Gang Sun, Kun Zhou, Wenjing Pang, Dachun Cao, Xin Tang and Xiangjun Meng

Correction to: Cell Death Discovery

https://doi.org/10.1038/s41420-021-00402-6

published online 19 January 2021

The original version of this article unfortunately contained a mistake. The last word of the third sentence in the abstract "OS patients" should be changed to "CRC patients". The authors apologize for the mistake. The original article has been corrected.

Published online: 21 October 2021 